\title{
ON A COMBINATORIAL PROBLEM ASSOCIATED WITH THE ODD ORDER THEOREM
}

\author{
GEORGE GLAUBERMAN AND SIMON P. NORTON
}

(Communicated by Ronald M. Solomon)

\begin{abstract}
We prove a conjecture about finite fields that arose in Péterfalvi's study of the Feit-Thompson Theorem.
\end{abstract}

\section{INTRODUCTION}

Let $p$ and $q$ be primes, and set

$$
\begin{gathered}
F_{p}=\mathrm{GF}(p), \quad F=\mathrm{GF}\left(p^{q}\right), \quad F^{*}=F-\{0\}, \quad U=\left\{b^{p-1} \mid b \in F^{*}\right\}, \\
E=\{b \in U \mid 2-b \in U\}, \quad \text { and } E^{-1}=\left\{b^{-1} \mid b \in E\right\} .
\end{gathered}
$$

Let $N$ be the norm function from $F$ into $F_{p}$. Since $F^{*}$ is a cyclic group under multiplication, it is easy to see that $U$ is a subgroup of $F^{*}$ and consists of all $b \in F$ for which

$$
1=b^{\left(p^{q}-1\right) /(p-1)}=b b^{p} b^{p^{2}} \cdots b^{p^{q-1}}=N(b) .
$$

In a simplification [3] of the last chapter of the proof of the Feit-Thompson odd order theorem [1], Péterfalvi proves the following results (their statements have been generalized somewhat, since no change in the proofs is required):

Lemma 1. Suppose $E=E^{-1}$ and $|E| \geq 2$. Then

(a) for each $b \in E$ and $k \in \mathrm{GF}(p), 1+k(1-b) \in U$, and

(b) $p \leq q$.

Lemma 2. If $p$ and $q$ are odd, then $|E| \geq 2$.

(Note that $|E| \geq 1$ because $1 \in E$.)

Lemma 3. Given the existence of a group satisfying certain restrictions (i.e., the hypothesis of Proposition 9), $E=E^{-1}$.

The proofs of Lemmas 1 and 2 are fairly short and based on Lemmas 38.938.11 of [1]; the bulk of [3] is occupied with the proof of Lemma 3. In the chapters of [1] preceding the last chapter, the authors prove that for any minimal simple group $G$ of odd order, there are distinct odd primes $p$ and $q$ such that, for both $(p, q)$ and $(q, p)$, the restrictions in Lemma 3 are satisfied by some

Received by the editors April 13, 1992.

1991 Mathematics Subject Classification. Primary 11T06; Secondary 20D05. 
subgroup of $G$. Therefore, these lemmas yield a contradiction, which completes the proof of the solubility of any finite group of odd order.

It is easy to show (Lemma 5) that Lemma 2 remains valid if $p=2$. In private communication, Peterfalvi conjectured that if $E=E^{-1}$ then $p \leq 3$. The main result of this paper is to prove this conjecture (Proposition 7). Although this does not appear to simplify further the proof of [1], it may be of some interest. We also discuss a related open question in $\S 3$.

Lemma 4. (a) If $p=2$, then $U=E=E^{-1}$.

(b) If $p=3$, then $E=E^{-1}$.

Proof. Since (a) is trivial, we prove (b). Assume $p=3$ and $c \in E$. Writing $N$ for the norm function from $F$ into $F_{p}$ (as before), we have

$$
N(c)=N(2-c)=1 \text {. }
$$

Therefore, $N\left(c^{-1}\right)=1$. Since $2-c^{-1}=c^{-1}(2 c-1)=c^{-1}(2-c)$ (because $p=3$ ),

$$
N\left(2-c^{-1}\right)=N\left(c^{-1}\right) N(2-c)=1 .
$$

Thus, $c^{-1} \in E$, as desired.

Lemma 5. For any primes $p$ and $q$,

(a) $E=\{1\}$ if $p$ is odd and $q=2$, and

(b) $|E| \geq 2$ otherwise.

Proof. If $p=2$, then $|E|=|U| \geq 2$ by Lemma 4. If $p$ and $q$ are odd, then $|E| \geq 2$ by Lemma 2 .

Now we may assume that $P$ is odd and $q=2$. Let $N$ be the norm function from $F$ into $F_{p}$, and take any $b \in E$. Let $c=b+b^{p}$, and let $f(x)$ be the polynomial $(x-b)\left(x-b^{p}\right)$. Then

$$
1=N(b)=b b^{p}, \quad f(x)=x^{2}-\left(b+b^{p}\right) x+b b^{p}=x^{2}-c x+1,
$$

and

$$
1=N(2-b)=(2-b)\left(2-b^{p}\right)=f(2)=4-2 c+1 .
$$

Therefore,

$$
c=2, \quad f(x)=x^{2}-2 x+1=(x-1)^{2}, \quad \text { and } \quad b=1,
$$

as claimed.

\section{MAIN RESULts}

Here, we continue using the notation in (1) but also regard $F$ as an affine space over $F_{p}$.

Proposition 6. Let $A$ be an affine space of finite dimension $r$ over a field $F_{p}$ of prime order $p$. Let $V$ be a vector space over $F_{p}$ associated with $A$. Suppose a subset $S$ of $A$ satisfies the condition:

Whenever $b \in A, x \in V$, and $b-x, b, b+x \in S$, then $b+k x \in S$ for all $k \in F_{p}$.

Assume $p \geq 5$ and $|S| \geq \frac{1}{2}|A|$. Then $S=A$. 
Remark. Condition (C) asserts that any affine line intersecting $S$ in at least three points, one midway between two others, lies in $S$. It is trivially true if $p$ is 2 or 3 , which makes it easy to construct counterexamples to the proposition in these cases (e.g., with $|A|=2^{2}$ or 3 ).

Proof. We divide the proof into two cases.

Case 1. $r=1$. Here, we may assume that $A=F_{p}$. Let $m=\frac{1}{2}(p-1)$. Then $|S|>\frac{1}{2} p$. So

$$
|S| \geq \frac{1}{2}(p+1)=m+1 \geq 3 .
$$

Take distinct elements $b, c \in S$, and let

$$
S^{\prime}=\left\{(b-c)^{-1}(x-c) \mid x \in S\right\} .
$$

It is easy to see that all translations and nonzero scalar multiplications preserve condition (C). Hence, $S^{\prime}$ satisfies (C). Replacing $S$ by $S^{\prime}$, we may assume that $0,1 \in S$.

Now suppose that $S$ is a proper subset of $A$. We will obtain a contradiction. Let us identify the elements of $A$ with the integers $-m$, $-(m-1), \ldots,-1,0,1, \ldots, m$ in the obvious manner. For each integer $k$ such that $1 \leq k \leq m$, the set $S$ cannot contain the sequence $-k, 0, k$, because $S$ satisfies (C) and does not contain the line $A$. Hence, for each $k, S$ contains at most one of the elements $k,-k$. Since there are $m$ pairs $k,-k$, and since $|S-\{0\}|=|S|-1 \geq(m+1)-1=m$,

$S$ must contain precisely one element from each pair $k,-k$.

Now $0,1 \in S$. To avoid the sequence $0,1,2$, we must have $-2 \in S$. Thus,

$$
0,1,-2 \in S \text {. }
$$

If $p=5$, then $1+2=-2$ and $(-2)+2=0$, so that the sequence $b-$ $x, b, b+x$ lies in $S$ for $b=-2$ and $x=2$, a contradiction. Thus, $p \geq 7$.

If $p \geq 11$, then, by (2) and (3), $S$ contains one of the sequences $0,-2,-4$ or $-2,1,4$, a contradiction. But then $p=7$, and similarly $S$ contains one of the sequences $0,-2,3$ or $-2,1,-3$, again a contradiction.

Case 2. General case. Again we assume $S \neq A$ and work by contradiction. Take $\lambda \in A \backslash S$, and let $\mathscr{L}$ be the set of all lines of $A$ that contain $\lambda$. Let

$$
A^{\prime}=A-\{\lambda\} \text { and } L^{\prime}=L-\{\lambda\} \text { for each } L \in \mathscr{L} .
$$

Then $A^{\prime}$ is the disjoint union of the sets $L^{\prime}$.

For each $L \in \mathscr{L}$, Case 1 yields that $|L \cap S| \leq \frac{1}{2}|L|=\frac{p}{2}$, and hence

$$
\left|L^{\prime} \cap S\right|=|L \cap S| \leq \frac{1}{2}(p-1)=\frac{1}{2}\left|L^{\prime}\right| .
$$

Therefore,

$$
|S|=\left|A^{\prime} \cap S\right|=\sum_{L \in \mathscr{L}}\left|L^{\prime} \cap S\right| \leq \frac{1}{2} \sum_{L \in \mathscr{L}}\left|L^{\prime}\right|=\frac{1}{2}\left|A^{\prime}\right|<\frac{1}{2}|A|,
$$

contrary to hypothesis. This completes the proof of Case 2 and of Proposition 6.

Remark. We thank Curtis Bennett for a suggestion which shortened part of the proof above. 
Proposition 7. Let $p$ and $q$ be primes, and assume the notation of (1). Then $E=E^{-1}$ if and only if $p \leq 3$.

Proof. If $p \leq 3$, then $E=E^{-1}$ by Lemma 4 .

Assume $p \geq 5$ and $E=E^{-1}$. We shall obtain a contradiction. By Lemma 5 , we have

$$
E=E^{-1} \text { and }|E| \geq 2 .
$$

We regard $F$ as an affine space over $F_{p}$.

Step 1 . For every affine subspace $A$ of $F$ over $F_{p}$, condition (C) of Proposition 6 is satisfied for $S=A \cap U$.

Proof. Recall that, for each $A$ and each $b \in A, A=b+V$ for some vector subspace $V$ of $F$ over $F_{p}$.

Suppose $b \in A, x \in V$, and $b-x, b, b+x \in A \cap U$. Let $d=b^{-1} x$ and $c=1-d$. Since $U$ is a group under multiplication, $U$ contains $b^{-1}(b-x)$ and $b^{-1}(b+x)$; that is,

$$
c=1-d=b^{-1}(b-x) \in U \text { and } 2-c=1+d=b^{-1}(b+x) \in U .
$$

So $c \in E$. By (5) and Lemma 1 ,

$$
1+k d=1+k(1-c) \in U \text { for every } k \in F_{p} .
$$

Hence

$$
b+k x=b(1+k d) \in U \text { for every } k \in F_{p} .
$$

This verifies condition (C) for $S=A \cap U$ and completes the proof of Step 1 .

By (5), $E$ contains some element $c \neq 1$. Let $b=1-c$. Then $b \neq 0$. For each positive integer $r=1,2, \ldots, q$, let

$$
A_{r}=\left\{1+k_{1} b+k_{2} b^{2}+\cdots+k_{r} b^{r} \mid k_{1}, \ldots, k_{r} \in F_{p}\right\} .
$$

Then each $A_{r}$ is an affine subspace of $F$ over $F_{p}$.

Step 2. We have

$$
A_{1} \subseteq U \text { and }\left|A_{r}\right|=p^{r} \text { for } r=1,2, \ldots, q .
$$

Proof. By Lemma $1, A_{1} \subseteq U$. Since $b \neq 0$ and $0 \notin U$, this shows that $b \notin F_{p}$. Since $q$ is a prime and $b \in F=\operatorname{GF}\left(p^{q}\right)$,

$$
b \text { has degree } q \text { over } F_{p} \text {. }
$$

Now, for each $r=1, \ldots, q$, the affine space $A_{r}$ consists of all elements of the form

$$
\begin{aligned}
1+b f(b) \text { for } f \text { the zero polynomial or a polynomial } \\
\\
\text { over } F_{p} \text { of degree at most } q-1 .
\end{aligned}
$$

We claim that distinct polynomials give distinct elements. To see this, assume that $1+b f_{1}(b)=1+b f_{2}(b)$. Since $b \neq 0, f_{1}(b)=f_{2}(b)$ and $\left(f_{1}-f_{2}\right)(b)=0$. Since $b$ has degree $q$ and $f_{1}-f_{2}$ has degree at most $q-1$, it follows that $f_{1}=f_{2}$, as claimed. This shows that $\left|A_{r}\right|=p^{r}$ for $r=1,2, \ldots, q$, as desired, and completes the proof of Step 2.

Step 3. For $r=1,2, \ldots, q, A_{r} \subseteq U$. 
Proof. By Step 2, $A_{1} \subseteq U$. Suppose $2 \leq r \leq q$ and $A_{r-1} \subseteq U$. A typical element $c$ of $A_{r}$ has the form $c=1+b f(b)$ for some polynomial

$$
f(X)=k_{1}+k_{2} X+\cdots+k_{r} X^{r-1}
$$

over $F_{p}$. If $1+X f(x)$ has degree less than $r$, or is a reducible polynomial, then induction yields that $c \in U$ (since $U$ is closed under multiplication and any factors of $1+X f(X)$ may be assumed to have constant term equal to 1$)$. Otherwise, $1+X f(X)$ is irreducible of degree $r$; that is,

$$
X^{r}+k_{r}^{-1}\left(k_{r-1} X^{r-1}+\cdots+k_{1} X+1\right)
$$

is irreducible. Each such polynomial corresponds to a set of $r$ distinct roots of the form $\lambda, \lambda^{p}, \ldots, \lambda^{p^{r-1}}$ in the field $\mathrm{GF}\left(p^{r}\right)$. Now, each such root generates $\mathrm{GF}\left(p^{r}\right)$ over $F_{p}$ and thus lies in $\mathrm{GF}\left(p^{r}\right) \backslash F_{p}$. Hence, there are at most $\left(p^{r}-p\right) / r$ distinct such polynomials. Consequently,

$$
\begin{aligned}
\left|A_{r} \cap U\right| & \geq\left|A_{r}\right|-r^{-1}\left(p^{r}-p\right) \geq p^{r}-\frac{1}{2}\left(p^{r}-p\right) \\
& =\frac{1}{2} p^{r}+\frac{1}{2} p>\frac{1}{2} p^{r}=\frac{1}{2}\left|A_{r}\right| .
\end{aligned}
$$

Since $p \geq 5$, Step 1 and Proposition 6 yield that $A_{r} \subseteq U$. This completes the proof of Step 3.

Step 4. Contradiction.

Proof. By Steps 2 and 3,

$$
|F|=p^{q}=\left|A_{q}\right| \leq|U|=\left(p^{q}-1\right) /(p-1)<|F| .
$$

This shows Step 4 and completes the proof of Proposition 7.

\section{FURTHER QUESTIONS}

As in the previous sections we use the notation of (1) for arbitrary primes $p, q$. Proposition 7 asserts that $E=E^{-1}$ if and only if $p \leq 3$. Lemma 3 asserts that the existence of a group satisfying certain restrictions yields that $E=E^{-1}$ and hence, by Proposition 7, that $p \leq 3$. In this section, we will specify the restrictions and investigate whether such a group can actually occur.

Lemma 8. For any primes $p$ and $q,\left(p^{q}-1\right) /(p-1)$ is relatively prime to $p-1$ if and only if $q$ does not divide $p-1$.

Proof. Let $r=p-1$. Then, modulo $r$,

$$
\left(p^{q}-1\right) /(p-1) \equiv p^{q-1}+p^{q-2}+\cdots+1 \equiv q,
$$

which yields the result.

Lemma 8 allows us to restate the main result of [3] slightly differently:

Proposition 9 [3, Lemma 3]. Let $p$ and $q$ be arbitrary primes, and assume the notation of (1). Let $P$ be the additive group of $F$, and let $U$ act on $P$ by multiplication. Identify $P$ and $U$ with their images in the semidirect product $H=P U$. Let $P_{0}$ be the image of the additive group of $F_{p}$. Assume that:

(A) $q$ does not divide $p-1$; and 
(B) there exist a monomorphism $\sigma$ of $H$ into a group $G$, a finite abelian $p^{\prime}$-subgroup $Q$ of $G$, and an element $y \in Q$ such that $\sigma\left(P_{0}\right)$ normalizes $Q$ and $\sigma\left(P_{0}\right)^{y}$ normalizes $U$. Then $E=E^{-1}$.

As mentioned above, we must have $p \leq 3$ in the situation of Proposition 9. In a personal communication, Péterfalvi gave the following examples and problem.

Example 10 (for Proposition 9). Let $p=2$ and let $q$ be any prime. Take

$$
\begin{gathered}
G=\operatorname{SL}\left(2,2^{q}\right), \quad \sigma(P)=\left\{\left(\begin{array}{ll}
1 & a \\
0 & 1
\end{array}\right) \mid a \in F\right\}, \\
\sigma(U)=\left\{\left(\begin{array}{cc}
a & 0 \\
0 & a^{-1}
\end{array}\right) \mid a \in F \backslash\{0\}\right\}, \\
\sigma\left(P_{0}\right)=\left\langle\left(\begin{array}{ll}
1 & 1 \\
0 & 1
\end{array}\right)\right\rangle, \quad y=\left(\begin{array}{ll}
0 & 1 \\
1 & 1
\end{array}\right), \quad Q=\langle y\rangle .
\end{gathered}
$$

Example 11 (for Proposition 9). Let $p=2$ and let $q$ be any odd prime. Take $G=S z\left(2^{q}\right)$, and choose $\sigma$ so that $\sigma(P)=\Omega_{1}(S)$ for some Sylow 2-subgroup $S$ of $G$ and $\sigma(U)$ is a (cyclic) subgroup of order $2^{q}-1$ in $N_{G}(S)$. Let $P_{1}$ be any subgroup of order 2 in $N_{G}(U)$. Then

$$
\sigma\left(P_{0}\right)=\left\langle x_{0}\right\rangle \text { and } P_{1}=\left\langle x_{1}\right\rangle
$$

for some elements $x_{0}, x_{1}$ of order 2. Let $Q=\left\langle x_{0} x_{1}\right\rangle$.

Suppose $Q$ has even order. Then it contains a unique element $x_{2}$ of order 2 . Since $x_{0}$ and $x_{1}$ invert $Q$, they centralize $x_{2}$. From the structure of $S z\left(2^{q}\right)$ [2, §§XI.1, XI.3], $\Omega_{1}(S)$ is abelian and $S=C_{G}(x)$ for each $x \in \Omega_{1}(S)^{\sharp}$. Hence

$$
x_{2} \in C_{G}\left(x_{0}\right)=S, \quad x_{1} \in C_{G}\left(x_{2}\right)=S, \quad\left[x_{1}, U\right] \subseteq[S, U] \cap U \subseteq S \cap U=1 .
$$

But then $U \subseteq C_{G}\left(x_{1}\right)=S$, which is absurd. Thus, $Q$ has odd order.

Now, $\sigma\left(P_{0}\right)$ and $P_{1}$ are Sylow 2-subgroups of $\left\langle x_{0}, Q\right\rangle$, and $\sigma\left(P_{0}\right)^{y}=P_{1}$ for some $y \in Q$.

Problem (Péterfalvi). Can the hypothesis of Proposition 9 be satisfied for $p=$ 3 ?

Note that condition (A) forces $q$ to be odd and $\sigma\left(P_{0}\right)^{y}$ must centralize $\sigma(U)$ if $\mid$ Aut $U \mid$ is not divisible by 3 (e.g., if $q=5$ and $\left.|U|=\left(3^{5}-1\right) /(3-1)=11^{2}\right)$.

\section{REFERENCES}

1. W. Feit and J. G. Thompson, Solvability of groups of odd order, Pacific J. Math. 13 (1963), 775-1029.

2. B. Huppert and N. Blackburn, Finite groups. III, Springer-Verlag, Berlin, 1982.

3. T. Peterfalvi, Simplification du chapitre VI de l'article de Feit et Thompson sur les groupes d'ordre impair, C. R. Acad. Sci. Paris Sér. I Math. 229 (1984), 531-534.

Department of Mathematics, University of Chicago, 5734 S. University Avenue, Chicago, Illinois 60637

Department of Pure Mathematics and Mathematical Statistics, University of CamBRIDGe, 16 Mill LANe, CAMbridge CB 2 1SB, England

E-mail address: spn1@phx.cam.ac.uk 